\title{
Survival from a Triple Co-Infection of COVID-19, HIV, and Tuberculosis: A Case Report
}

\author{
Tadesse Tolossa (1D) \\ Reta Tsegaye (iD) ${ }^{2}$ \\ Siraj Shiferaw ${ }^{3}$ \\ Bizuneh Wakuma $\mathbb{D}^{2}$ \\ Diriba Ayala ${ }^{4}$ \\ Belay Bekele ${ }^{5}$ \\ Tesfaye Shibiru ${ }^{3}$ \\ 'Department of Public Health, Institute \\ of Health Sciences, Wollega University, \\ Nekemte, Ethiopia; ${ }^{2}$ Department of \\ Nursing, Institute of Health Sciences, \\ Wollega University, Nekemte, Ethiopia; \\ ${ }^{3}$ School of Medicine, Institute of Health \\ Sciences, Wollega University, Nekemte, \\ Ethiopia; ${ }^{4}$ Department of Midwifery, \\ Institute of Health Sciences, Wollega \\ University, Nekemte, Ethiopia; ${ }^{5}$ Wollega \\ University Referral Hospital, Institute of \\ Health Sciences, Wollega University, \\ Nekemte, Ethiopia
}

Introduction: The triple burden of COVID-19, human immune virus (HIV), and TB coinfection is one of the contemporary challenges to this globe. People living with chronic medical diseases such as HIV and TB are more likely to experience poor outcomes from COVID-19. This study aimed to report survival from triple co-infection with PTB, HIV, and COVID-19 in Wollega University referral hospital (WURH), western Ethiopia.

Case Presentation: A 55-year-old male known HIV patient on HAART for the last 3 years presented to WURH on May 26, 2021, with chief complaints of shortness of breath of 5 days' duration associated with productive cough, history of high-grade fever, and severe headache. He had produced a lot of bloody sputum one month prior to the current complaints. On 1st day of his presentation, a Gene Expert test was done from sputum, and he was positive for pulmonary TB. On the same day, a nasopharyngeal sample for COVID-19 using polymerase chain reaction (PCR) was positive. On his 2nd day of admission, he was transferred to the COVID-19 treatment center with the diagnosis of severe COVID-19, PTB, and WHO stage-3 RVI. During his stay in the hospital, he was provided with oxygen via a nasal cannula. Intensive phase anti-TB treatment was initiated on the 3rd day of admission. HAART was continued with the same regimen he was taking prior to his presentation. On June 6, 2021, the patient was recovered and discharged from the hospital after 10 days of hospital stay.

Conclusion: This case report suggests, after 10 days of admission with triple infections, there is a good clinical prognosis and survival from COVID-19. On admission, further diagnosis and investigations of TB co-infection should be performed for all patients admitted with COVID-19 due to their similar and non-specific clinical manifestations.

Keywords: co-infection, COVID-19, HIV, PTB, Ethiopia

\section{Introduction}

The triple burden of COVID-19, human immune virus (HIV), and tuberculosis (TB) co-infection is one of the contemporary challenges to this globe. ${ }^{1}$ COVID-19 is currently the 12th leading cause of death globally, and the 6th leading cause of death in developed countries. ${ }^{2}$ Globally, more than 173.3 million people had been infected and more than 3.7 million had died of coronavirus as of July 9, 2021, ${ }^{3}$ and the figures are still rising rapidly. ${ }^{4,5}$

The symptoms of COVID-19 range from mild (or no symptoms) to severe illness and are mainly characterized by fever, dry cough, dyspnea, headache, sore throat and rhinorrhea, and sometimes hemoptysis. ${ }^{6,7}$ Increasing numbers of severe forms of COVID-19 are expected to rapidly occur among patients who had been affected with chronic medical conditions like TB and HIV/AIDS. ${ }^{8,9}$ As a result,
Correspondence: Tadesse Tolossa

Department of Public Health, Institutes of Health Sciences, Wollega University, P.O.BOX: 395, Nekemte, Ethiopia Tel +25I9II936804

Email yadanotolasa@gmail.com 
COVID-19 patients with coexisting medical illnesses like TB and HIV/AIDS are believed to experience poor treatment outcome from COVID-19. ${ }^{10}$ Patients living with HIV might be at higher risk of developing COVID-19related complications and poor clinical outcomes due to various reasons like a weak immune system, presence of several co-infections, and side effect of ART drug. ${ }^{10,11}$ Furthermore, the diagnosis and management of TB and COVID-19 co-infection are challenging due to the similarity of clinical features, which may lead to misdiagnosis of the diseases. A pre-existing TB and other lung diseases increase the severity of the COVID-19. In addition, COVID-19 needs immune activator medication which may lead to reactivation of latent $T B$, and also there is a possibility of drug-drug interaction among anti-TB drugs and medicines which are used for COVID-19 cases. $^{12}$

A couple of studies have been conducted on recovery from triple co-infection in developed countries, ${ }^{13-15}$ but there are no reports of COVID-19 and TB co-infections in previously known HIV-positive patients on ART in SSA including Ethiopia. We describe the survival of one confirmed COVID-19 case co-infected with TB in a previously known HIV-positive patient on ART in WURH treatment center, western Ethiopia.

\section{Case Finding}

A 55-year-old male known HIV-positive patient on antiretroviral therapy (TDF $300 \mathrm{mg}+$ FTC $200 \mathrm{mg}+\mathrm{EFV}$ $600 \mathrm{mg}$ ) for the last 3 years was referred from Gidda Ayana District Hospital on May 26, 2021 to Wollega University referral hospital. Upon arrival to the emergency department, he complained of shortness of breath of five days' duration, which began suddenly, and became more and more persistent, associated with productive cough, high-grade fever, malaise, and severe headache. In addition, he had a loss of appetite, weight loss, chest pain, night sweats, and fatigue. Moreover, he had a history of bloody sputum one month prior to the current complaint. He has tolerated well but had poor adherence to his ART medication and had no previous history of alcohol consumption and other illicit drugs. He has reported receiving cotrimoxazole prophylaxis for three years, but often missed some doses. His current CD4 count was 266 cells $/ \mathrm{mm}^{3}$, and HIV-1 RNA level was 9000 copies/mL.

Upon physical examination at presentation, the patient looked acutely sick, and blood pressure was measured at $110 / 60 \mathrm{mmHg}$, a body temperature of $39.3^{\circ} \mathrm{C}$, and pulse rate of 112 beats per minute, classed as tachycardia. His respiratory rate was 38/minute, and his oxygen saturation was $87 \%$ on room air. His breathing was shallow, rapid, and used respiratory accessory muscles. There was dullness over the left lower lobe. Breathing sounds were vesicular on the right side but markedly reduced at the base. There was coarse crepitation over the left upper and lower lobes. He was conscious and oriented. Immediately the patient was admitted as a medical emergency and put on intranasal oxygen.

Necessary laboratory investigations were performed. Accordingly, random blood sugar was $110 \mathrm{mg} / \mathrm{dL}$, complete blood count with differential revealed only leukocytosis $\left(15.2 \times 10^{3} / \mathrm{L}\right)$ with lymphocyte predominance, erythrocyte sedimentation rate (ESR) of $86 \mathrm{~mm} /$ hour; blood film $(\mathrm{BF})$ showed no hemoparasite, serum creatinine was $0.9 \mathrm{mg} / \mathrm{dL}$, and liver function test showed no abnormal value. Chest X-ray showed perihilar patchy opacities (Figure 1).

Gene Expert test was done from sputum and was positive for TB (no rifampin resistance), and nasopharyngeal sample for SARS CoV-2 PCR was positive. On his 2nd day of admission, he was transferred to a COVID-19 dedicated treatment center located in the same hospital with the diagnosis of severe COVID-19, pulmonary TB, and WHO stage-3 RVI.

During his stay, oxygen supplementation for an $\mathrm{SpO}_{2}$ target $\geq 92-96 \%$ was provided with $6-10 \mathrm{~L} \mathrm{O}_{2}$ via a nasal cannula. Conservative IV fluid management with empiric antimicrobials for possible bacterial infection was started with cefepime $2 \mathrm{~g}$ IV TID for 3 days then changed to an oral antibiotic. In addition, dexamethasone $6 \mathrm{mg}$ once per day IV was given with close monitoring of vital signs, work of breathing, and mental status. Anti-TB drugs were initiated with RHZE (rifampicin, isoniazid, pyrazinamide, and ethambutol) at the intensive phase on the 3rd day of admission. Highly active antiretroviral therapy was continued with the same regimen he was taking before presentation to the hospital.

On his 6th day of admission, the patient became afebrile and started to maintain his oxygen saturation $>94 \%$ with room air. The patient's general condition also improved. Follow-up RT-PCR test for COVID-19 was done, and the results were negative. On June 6, 2021 the patient was discharged from hospital after 10 days of hospital stay, and a follow-up appointment at the ART and TB clinic within two weeks was given.

\section{Discussion}

This case report was intended to discuss the triple coinfection of TB, HIV, and COVID-19 diagnosis, and the 


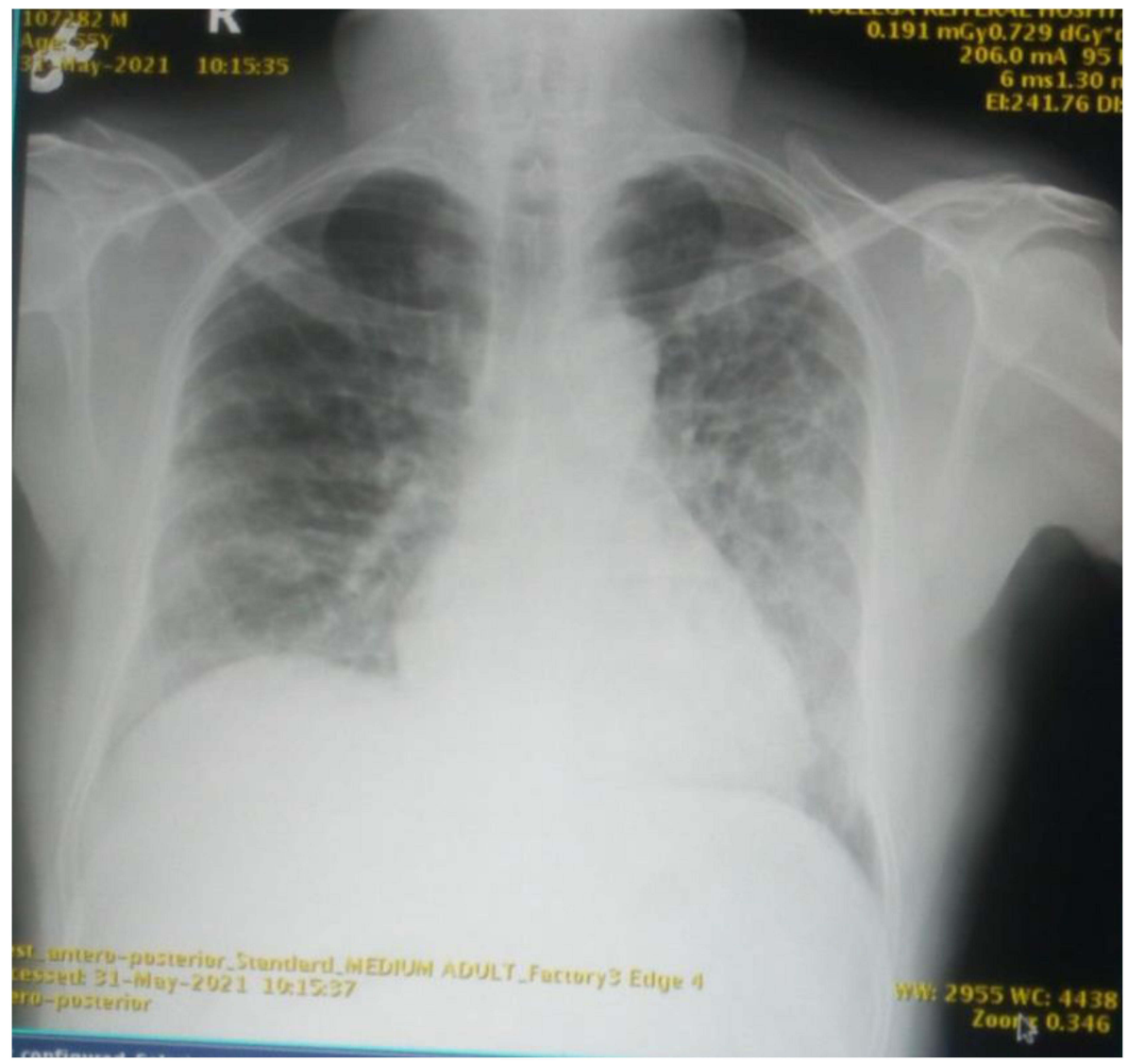

Figure I Chest X-ray result showing perihilar patchy opacities.

treatment and outcome of a single patient. In this case study, the patient admitted with triple co-infections of HIV/AIDS, TB, and COVID-19 recovered after 10 days of hospital stay. Although COVID-19 affects all types of individuals, patients with older age, multiple comorbidities including advanced chronic obstructive lung disease, and associated cardiovascular illnesses are reported to be at risk of the worst outcome. ${ }^{16}$

COVID-19 is a contagious disease with recurrent and severe pulmonary involvement, which can lead to viral pneumonia, hospitalizations, and deaths. According to one meta-analysis study, HIV/TB co-infection increases the risk of COVID-19 severity and the mortality. Additionally, the HIV/TB co-infected group has the highest risk in the COVID-19 mortality rate and poor recovery rate. $^{17,18}$ In a patient with multiple co-infections with COVID-19, PTB, and HIV, the clinical outcome is expected to be worsened. However, in this case report, gradual recovery from COVID-19 was observed after treatment with oxygen supplementation, dexamethasone, and antibiotics, besides the usual anti-TB and ARV medications. Similar recovery from COVID-19 was observed in similar cases reported in Brazil and in Panama. ${ }^{14,18}$ This might be attributed to the strict adherence to ART and anti-TB medications initiated after admission in the current case report, though further research is still needed to justify this.

The few available data on interactions between COVID-19, TB, and HIV have revealed that immunosuppressed PLHIV with low CD4+ cell count and high viral loads are at high risk for acute respiratory distress syndrome in other viral co-infections; the study found that people living with HIV had almost tripled the risk of dying from COVID-19 compared to those without HIV. ${ }^{19}$ However, other literatures found out that coinfection with HIV and COVID-19 does not appear to worsen COVID-19 infection outcomes. The greatest number of complicated COVID-19 cases occur among patients with comorbid chronic diseases, such as diabetes, hypertension, and cancer, and being older than 60 years. However, the evidence is not sufficient to indicate whether mortality in patients with a severe 
immunosuppressed state is higher or lower. ${ }^{14}$ In this regard, being HIV-positive did not hinder our patient to recover from COVID-19.

\section{Limitations}

According to the knowledge of authors, there is no published article on the survival status of HIV-positive patients admitted with COVID-19 and TB co-infections. However, this study has a couple of limitations; the study is a case report done on a single case, and therefore results may not be generalizable to the general populations.

\section{Conclusions}

In general, co-infection of COVID-19 and TB both in general population and patients on ART is becoming common in SSA, where the prevalence of HIV and TB is high. On admission, further diagnosis and investigations of TB co-infection should be performed for all patients admitted with COVID-19 due to their similar and non-specific clinical manifestations. Finally, our study recommends that comprehensive diagnosis of patients with COVID-19, combined with proper clinical and therapeutic management with psychological support, will lead to good prognosis of patients with triple co-infections. A larger study with large sample size will be required to validate findings from this study.

\section{Abbreviations}

ART, antiretroviral therapy; HIV, human immune virus; EFV, efavirenz; FTC, emtricitabine; IV, intravenous; RT$\mathrm{PCR}$, reverse transcription polymerase chain reaction; RVI, retroviral infection; SSA, sub-Saharan Africa; TID, three times a day; TB, tuberculosis; WHO, World Health Organization; TDF, tenofovir.

\section{Ethical Approval and Consent to Participate}

The study was approved and ethical clearance letters were obtained from Wollega University, Institute of Health Science research Ethics review board. After approval, a permission letter was obtained from the administrative body of WURH to take and revise history of patient. A written informed consent was obtained from the study participant, and the purpose of this study was stated to the patient.

\section{Consent to Publish}

A written informed consent was obtained from the study participant, and the purpose of this study was stated to the patient.

\section{Author Contributions}

SS and TS wrote and organized history of the patient. TT, $\mathrm{RT}$, and BW were involved in the initial development the manuscript. All authors contributed to data analysis, drafting, or revising the article, have participated in the final preparation of manuscript, have agreed on the journal to which the article will be submitted, gave final approval of the version to be published, and agree to be accountable for all aspects of the work.

\section{Funding}

No funding was obtained for this study.

\section{Disclosure}

The authors declare that they have no competing interests.

\section{References}

1. Sarkar S, Khanna P, Singh AK. Impact of COVID-19 in patients with concurrent co-infections: a systematic review and meta-analyses. J Med Virol. 2021;93(4):2385-2395. doi:10.1002/jmv.26740

2. Kirenga BJ, Byakika-Kibwika P. Excess COVID-19 mortality among critically ill patients in Africa. Lancet. 2021;397(10288):1860-1861. doi:10.1016/S0140-6736(21)00576-6

3. Roy D, Tripathy S, Kar SK, et al. Study of knowledge, attitude, anxiety \& perceived mental healthcare need in Indian population during COVID-19 pandemic. Asian J Psychiatr. 2020;51:102083. doi:10.1016/j.ajp.2020.102083

4. Available from: https://www.worldometers.info/coronavirus/country/ ethiopia/. Accessed August 25, 2021

5. Shereen MA, Khan S, Kazmi A, et al. COVID-19 infection: origin, transmission, and characteristics of human coronaviruses. $J$ Adv Res. 2020;24:91-98. doi:10.1016/j.jare.2020.03.005

6. Tsai P-H, Lai W-Y, Lin -Y-Y, et al. Clinical manifestation and disease progression in COVID-19 infection. J Chinese Med Assoc. 2021;84 (1):3-8. doi:10.1097/JCMA.0000000000000463

7. Adhikari SP, Meng S, Wu Y-J, et al. Epidemiology, causes, clinical manifestation and diagnosis, prevention and control of coronavirus disease (COVID-19) during the early outbreak period: a scoping review. Infectious Dis Poverty. 2020;9(1):1-12. doi:10.1186/s40249020-00646-x

8. Härter G, Spinner CD, Roider J, et al. COVID-19 in people living with human immunodeficiency virus: a case series of 33 patients. Infection. 2020;1.

9. World Health Organisation. Coronavirus. Available from: https:// www.who.int/health-topics/coronavirus\#tab=tab_1. Accessed August 25, 2021. 2020.

10. Nagarakanti SR, Okoh AK, Grinberg S, et al. Clinical outcomes of patients with COVID-19 and HIV coinfection. J Med Virol. 2021;93 (3):1687-1693. doi:10.1002/jmv.26533

11. Aberg JA. Aging, inflammation, and HIV infection. Top Antivir Med. 2012;20(3):101. 
12. Kumar R, Bhattacharya DB, Meena DV, et al. COVID-19 and TB coinfection-'Finishing touch" in perfect recipe to 'severity'or 'death'. J Infection. 2020;81(3):e39-e40. doi:10.1016/j.jinf.2020.06.062

13. Ortiz-Martínez Y, Mogollón-Vargas JM, López-Rodríguez M, et al. A fatal case of triple coinfection: COVID-19, HIV and Tuberculosis. Travel Med Infect Dis. 2021;43:102129. doi:10.1016/j. tmaid.2021.102129

14. Rivas N, Espinoza M, Loban A, et al. Case report: COVID-19 recovery from triple infection with Mycobacterium tuberculosis, HIV, and SARS-CoV-2. Am J Trop Med Hyg. 2020;103(4):1597.

15. González-Domenech CM, Pérez-Hernández I, Gómez-Ayerbe C, et al. A pandemic within other pandemics. when a multiple infection of a host occurs: SARS-CoV-2, HIV and Mycobacterium tuberculosis. Viruses. 2021;13(5):931. doi:10.3390/v13050931

16. Mishra A, George AA, Sahu KK, et al. Tuberculosis and COVID-19 Co-infection: an Updated Review. Acta Biomed. 2020;92(1): e2021025.
17. Tamuzi JL, Ayele BT, Shumba CS, et al. Implications of COVID-19 in high burden countries for HIV/TB: a systematic review of evidence. BMC Infect Dis. 2020;20(1):744. doi:10.1186/s12879020-05450-4

18. Gadelha Farias LAB, Gomes Moreira AL, Austregésilo Corrêa E, et al. Case Report: coronavirus Disease and Pulmonary Tuberculosis in Patients with Human Immunodeficiency Virus: report of Two Cases. Am J Trop Med Hyg. 2020;103(4):1593-1596. doi:10.4269/ ajtmh.20-0737

19. Nachega JB, Kapata N, Sam-Agudu NA, et al. Minimizing the impact of the triple burden of COVID-19, tuberculosis and HIV on health services in sub-Saharan Africa. Int J Infect Dis. 2021. doi:10.1016/j. ijid.2021.03.038

\section{Publish your work in this journal}

The International Medical Case Reports Journal is an international, peer-reviewed open-access journal publishing original case reports from all medical specialties. Previously unpublished medical posters are also accepted relating to any area of clinical or preclinica science. Submissions should not normally exceed 2,000 words or 4 published pages including figures, diagrams and references. The manuscript management system is completely online and includes a very quick and fair peer-review system, which is all easy to use. Visit http://www.dovepress.com/testimonials.php to read real quotes from published authors. 